\title{
Serum Proteomic Patterns Associated With Sleep-Disordered Breathing in Children
}

\author{
ZAHOOR A. SHAH, SAEED A. JORTANI, RIVA TAUMAN, ROLAND VALDES, JR., AND DAVID GOZAL \\ Departments of Pediatrics [Z.A.S., R.T., D.G.], Pathology and Laboratory Medicine [S.A.J., R.V.], and Pharmacology and Toxicology \\ [D.G.], University of Louisville, Louisville, Kentucky 40202
}

\begin{abstract}
Obstructive sleep apnea (OSA) is a major public health problem affecting approximately $2 \%$ to $3 \%$ of children. However, snoring, the cardinal symptom of OSA, affects at least 5-fold more children, such that evaluation by overnight polysomnography (ONP) is required for the diagnosis. ONP is laborious, expensive, and relatively unavailable to children. Proteomic mass spectrometry coupled with bioinformatic tools provide valuable means for discovery of new biomarkers in serum for a variety of human disorders. The possibility exists that snoring children with and without OSA may exhibit different protein expression profiles in serum that could be useful in the development of novel diagnostic tools for this condition. The proteomic patterns of 20 children with OSA and of 20 children with habitual primary snoring but no evidence of OSA (HS) were evaluated using surface-enhanced laser desorption/ionization timeof-flight mass spectrometry (SELDI-TOF MS). Linear discriminative analysis identified three differentially regulated proteins with molecular masses of 5896, 3306, $6068 \mathrm{Da}$ that were capable of diagnosing OSA with $93 \%$ sensitivity and $90 \%$ specificity. Thus, the proteomic signatures of sera from children with OSA differ from those of HS who do not fulfill the current criteria for treatment. Identification and sequencing of those differentially expressed proteins discovered through proteomic strategies may lead to future development of serum-based diagnostic tests for OSA in snoring children. (Pediatr Res 59: 466-470, 2006)
\end{abstract}

$\mathrm{O}$ SA is a frequent condition affecting up to $2 \%$ to $3 \%$ of all children and is associated with substantial neurobehavioral and cardiovascular morbidities (1-3). In the United States alone, it is estimated that two million children suffer from OSA. However, many more children (8\%-27\%) habitually snore during their sleep and are at risk of OSA $(1,2,4-$ 8). Clinical history and physical examination are remarkably unpredictive in the differentiation between OSA and HS (9) such that current diagnostic approaches require implementation of ONP. However, ONP is laborious, expensive, and relatively unavailable to children. Furthermore, ONP imposes a substantial burden to both child and family.

Proteomic mass spectrometry coupled with advanced bioinformatic techniques has recently been introduced as a novel approach for developing new diagnostic and prognostic tools

Received March 21, 2005; accepted October 10, 2005.

Correspondence: David Gozal, M.D., Kosair Children's Hospital Research Institute, University of Louisville School of Medicine, 571 S. Preston Street, Suite 321, Louisville, KY 40202; e-mail: david.gozal@louisville.edu.

This study was supported in part by National Institutes of Health grant HL 65270 and The Commonwealth of Kentucky Research Challenge Trust Fund. for various diseases $(10,11)$. SELDI-TOF MS, which is based on the capture of proteins/peptides on chemically modified surfaces, has emerged as a specifically powerful tool allowing analysis of complex biologic samples $(12,13)$. For example, SELDI-TOF MS has been successfully used in the analysis of serum proteins to reveal disease-specific "fingerprints" or "patterns" that have proven useful in the early-stage detection of particular diseases due to its high specificity and sensitivity in segregating diseased patients from those without disease (14-16). Furthermore, the feasibility of high sample throughput and low volume sample requirements make this approach particularly valuable for discovery of new disease-specific biomarkers in children.

In this study, we used SELDI-TOF MS strategies and examined sera of 20 snoring children with OSA and of 20 snoring children who did not fulfill the polysomnographic criteria for OSA (i.e. HS).

\section{MATERIALS AND METHODS}

Study participants. The study was approved by the University of Louisville Human Research Committee. Parental informed consent and child assent, in the presence of a parent, were obtained. Children were excluded if they had any chronic medical condition, psychiatric diagnoses, or any genetic or craniofacial syndromes.

Morning serum samples were obtained from 20 consecutive snoring children fulfilling the criteria for OSA (see below), and from 20 consecutive children fulfilling the criteria for habitual snoring (HS). Their ages ranged between 3 and $12 \mathrm{y}$, and all were referred for evaluation of snoring as their primary complaint and therefore underwent an overnight sleep study at the Kosair Childen's Hospital Sleep Medicine and Apnea Center. Based on their polysomnographic findings, children were assigned to one of two categorical groups: group OSA, 20 children with an obstructive apnea/hypopnea index (AHI) $>5$ events/h of sleep, combined with oxyhemoglobin desaturation episodes $(<92 \%)$ and/or hypercapnia $(>50 \mathrm{~mm} \mathrm{Hg})$, and/or respiratory arousal index $>2$ /hour of sleep and group HS, 20 children with AHI $<1 / \mathrm{h}$ of sleep without oxyhemoglobin desaturation and/or hypercapnia, and with a respiratory arousal index $\leq 2 / \mathrm{h}$ of sleep.

Overnight polysomnography. A standard overnight multichannel polysomnographic evaluation was performed in the sleep laboratory at Kosair Children's Hospital. Children were studied for up to $12 \mathrm{~h}$ in a quiet, darkened room with an ambient temperature of $24^{\circ} \mathrm{C}$ in the company of one of their parents. No drugs were used to induce sleep. The following parameters were measured: chest and abdominal wall movement by respiratory impedance or inductance plethysmography and heart rate by electrocardiography; air flow

Abbreviations: AHI, obstructive apnea/hypopnea index; HS, primary habitual snorers; ONP, overnight polysomnography; OSA, obstructive sleep apnea; REM, rapid eye movement; SELDI-TOF MS, surface-enhanced laser desorption/ionization time-of-flight mass spectrometry; TST, total sleep time 
was monitored with a sidestream end-tidal capnograph that also provided breath-by-breath assessment of end-tidal carbon dioxide levels $\left(\mathrm{PeTCO}_{2} ; \mathrm{BCI}\right.$ SC-300, Menomonee Falls, WI), a nasal pressure transducer, and a thermistor. Arterial oxygen saturation $\left(\mathrm{SpO}_{2}\right)$ was assessed by pulse oximetry (Nellcor $\mathrm{N}$ 100; Nellcor Inc., Hayward, CA), with simultaneous recording of the pulse waveform. The bilateral electro-oculography, eight-channel electroencephalography, chin and anterior tibial electromyography, and analog output from a body position sensor (Braebon Medical Corporation, NY) were also monitored. All measures were digitized using a commercially available polysomnography system (Rembrandt, MedCare Diagnostics, Amsterdam, The Netherlands). Tracheal sound was monitored with a microphone sensor (Sleepmate, VA) and a digital time-synchronized video recording was performed. Sleep architecture was assessed by standard techniques (17). The proportion of time spent in rapid eye movement (REM) sleep was expressed as the percentage of total sleep time (TST). The obstructive apnea index (AI) was defined as the number of apneas episodes per hour of TST. Obstructive apnea was defined as the absence of airflow with continued chest wall and abdominal movement for duration of at least two breaths $(18,19)$. Hypopnea was defined as a decrease in nasal flow of $\geq 50 \%$ with a corresponding decrease in $\mathrm{SpO}_{2}$ of $\geq 4 \%$ and/or arousal (19). The apnea/hypopnea index (AHI) was defined as the number of apnea and hypopnea episodes per hour of TST. Children with an AHI $<1 / \mathrm{h}$ TST were considered to have habitual snoring (HS), while children with AHI $\geq 5 / \mathrm{h}$ TST were considered to have OSA. The mean oxygen saturation, as measured by pulse oximetry $\left(\mathrm{SpO}_{2}\right)$ in the presence of a pulse waveform signal void of motion artifact, and the $\mathrm{SpO}_{2}$ nadir were recorded. Since criteria for arousals have not yet been developed for children, arousals were defined as recommended by the American Sleep Disorders Association Task Force report (20) using the 3-s rule and/or the presence of movement arousal (21). Arousals were divided into two subtypes: spontaneous arousals and respiratory arousals. The arousal indices were calculated per hour of sleep time.

SELDI protein profiling of serum. Four chip types including weak cation exchange (WCX4) with low stringency ( $\mathrm{pH} 4)$, metal binding (IMAC-Cu ${ }^{2+}$ ), immobilized metal affinity capture, strong cation exchange (SAX2) and hydrophobic $(\mathrm{H} 4)$ chips were used to profile the serum samples collected from the OSA and HS children. We used a modified analytical protocol provided by the manufacturer (Ciphergen Biosystems, Fremont, CA) for chip activation, equilibration, binding, washing, and reading.

Chip activation. IMAC chips were activated by addition of $\mathrm{CuSO}_{4}$. This was done by adding $50 \mu \mathrm{L}$ of $100 \mathrm{mmol} / \mathrm{L} \mathrm{CuSO}_{4}$ in a 96-well formatted Bioprocessor (Ciphergen Biosystems) for $5 \mathrm{~min}$ at room temperature. The unbound $\mathrm{Cu}^{2+}$ was then removed; chips were rinsed by $\mathrm{dH}_{2} \mathrm{O}$ and 100 $\mathrm{mmol} / \mathrm{L} \mathrm{Na}$ acetate $(\mathrm{pH} 4.0)$. Other chip types did not require activation.

Chip equilibration. All chip types were equilibrated with their perspective binding/washing buffers according to manufacturer's instructions. Appropriate buffers for a given chip type $(150 \mu \mathrm{L})$ was added to each chip type (IMAC-Cu 2 ${ }^{+} ; 100 \mathrm{mmol} / \mathrm{L} \mathrm{Na}$ phosphate, $\mathrm{pH} 7.0+500 \mathrm{mmol} / \mathrm{L} \mathrm{NaCl}$, WCX4; $100 \mathrm{mmol} / \mathrm{L} \mathrm{Na}$ acetate, $\mathrm{pH}$ 4.0, SAX2; $10 \mathrm{mmol} / \mathrm{L}$ Tris, $\mathrm{pH}$ 8.0, H4; $50 \mathrm{mmol} / \mathrm{L}$ HEPES, $\mathrm{pH}$ 7.0) in a 96-well Bioprocessor and incubated for 5 min. Then, the buffer was removed by dumping in collection trays.

Protein binding. After thawing, the serum samples were mixed and centrifuged at $1000 \mathrm{rpm}$ at $4^{\circ} \mathrm{C}$ for $10 \mathrm{~min}$. Twenty microliters of serum was added into the 96 -well plate containing mixture of $30 \mu \mathrm{L}$ of $8 \mathrm{~mol} / \mathrm{L}$ urea, $1 \%$ CHAPS/PBS (pH 7.4), sealed and subjected to shake at $4^{\circ} \mathrm{C}$ for $15-30 \mathrm{~min}$.

Chip binding. In an assembled 96-well Bioprocessor containing the appropriate chips, $5 \mu \mathrm{L}$ of fractionated serum samples were added to $195 \mu \mathrm{L}$ of binding/washing buffer.

Chip washing. All chip arrays were first incubated in their respective binding/washing buffers for $5 \mathrm{~min}$ and buffers removed, which was repeated three times. Finally, chips were washed twice by adding $200 \mu \mathrm{L}$ of $\mathrm{dH}_{2} \mathrm{O}$, followed by dissembling the bioprocessor and chip drying with the help of Kim wipes. Spots on chips were circled with a PAP pen and $1 \mu \mathrm{L}$ of SPA as EAMs (electron absorbing molecules) was added twice.

Chip reading. We used two protocols developed in our laboratory for lowand high-mass focusing to read each chip. The low focusing has a mass range from 1000 to $50,000 \mathrm{Da}$ with optimization at 3000 to $30,000 \mathrm{Da}$. The laser intensity will be set at 285 and the instrument sensitivity at 9. For the high-mass focusing, we set the high threshold at 200,000 Da with an optimization range of 10,000 to $60,000 \mathrm{Da}$ and a laser intensity of 290 with a sensitivity setting of 9 .

Peak detection/normalization. All spectra were initially visually inspected. If the matrix peaks were missing or depressed below a 30\% log normalized value, the spectra were eliminated from final data analysis. All spectra were calibrated and normalized according to the manufacturer's instructions using the Biomarker Wizard Software (Ciphergen Biosystems). The peaks were detected using the same software and a ".csv" output was generated for each experiment using this software. The unsupervised quali- tative inspection of peaks and data clustering were performed at this stage Then, the ".csv" files were imported into Biomarker Pattern Software (BPS) software for data mining analysis.

Decision tree and cross-validation. We used linear discriminative analysis using BPS (Ciphergen Biosystems), which allows for supervised classification analysis capable of varying splitting rules, cost values, and creation of decision trees. BPS is designed to analyze large data sets and discover protein patterns that can be related to treatment and/or to pathology due to treatment. In this study, we considered a biomarker pattern as potentially useful if HS and OSA patients were distinguished with a sensitivity and specificity $>90 \%$. Decision tree classifies a spectrum pattern through a sequence of questions, in which the next question asked depends on the answer to the previous question. The sequence of questions forms a node connected by successive links or branches downward to other nodes.

Protein identification. Preliminary identification of the discovered proteins using the isoelectric point (pI) and molecular mass information was performed using the Swiss-Prot database.

\section{RESULTS}

Subject characteristics. Table 1 shows the demographic and polysomnographic characteristics of 20 children with sleep disordered breathing (OSA), and 20 HS children. Both groups were comparable for age, gender, and body mass index. The OSA group had higher mean AHI and arousal index, with lower $\mathrm{SaO}_{2}$ nadir, and similar mean end-tidal $\mathrm{CO}_{2}$ tension (Table 1).

Decision tree/data analysis. The mass range of 0-2000 Da was eliminated from data analysis because of interference by matrix peaks. To identify serum biomarkers capable of distinguishing the children with OSA from HS but no evidence of OSA, we constructed a decision-tree classification algorithm using over 100 peaks within the 2000- to 40,000-Da range. The classification algorithm was developed using the crossvalidation technique included in the BPS software and involved three nodes with distinct masses $(5896,3306,6068$ Da) as shown in Figure $1 A$ and $B$. The discriminatory peaks were able to efficiently distinguish 17 of 18 (94\%) children with OSA and 20 of $20(100 \%)$ HS without OSA (Table 2). In the test group of the cross-validation, 15 of $18(83 \%)$ OSA children and 18 of 20 (90\%) non-OSA snorers were identified (Table 2). Figure $2 A$ and $B$ represent the spectra and gel view of OSA and HS set of samples, respectively. A peak at 5896 $\mathrm{Da}$ is clearly observed in the OSA set of samples. On the other hand, the same peak was either absent or clearly attenuated in the HS group. The biomarker protein at the putative mass of $5896 \mathrm{Da}$ with stringency wash at pH.4 was subjected to the

Table 1. Demographic and polysomnographic characteristics in children with habitual snoring and children with OSA syndrome

\begin{tabular}{lcc}
\hline & \multicolumn{1}{c}{ OSAS } & HS \\
\hline Age $(\mathrm{y})$ & $8.5 \pm 3.7(3-16)$ & $8.7 \pm 3.8(5-17)$ \\
Sex $(\mathrm{male} \%)$ & 45 & 60 \\
$\mathrm{BMI}\left(\mathrm{kg} / \mathrm{m}^{2}\right)$ & $24.7 \pm 8.8(14.5-47.7)$ & $24.9 \pm 11.9(15.6-66)$ \\
$\mathrm{OAHI}(/ \mathrm{hr} \mathrm{TST})$ & $23.5 \pm 19.5(7.3-83)$ & $1.9 \pm 1.3(0.39-4.76)$ \\
$\mathrm{SpO}_{2}$ nadir $(\%)$ & $75.4 \pm 13.4(47-91)$ & $89.9 \pm 4.0 *(81-96)$ \\
$\mathrm{Mean} \mathrm{PEtCO}_{2}(\mathrm{~mm} \mathrm{Hg})$ & $49.3 \pm 7.4(37-64)$ & $47 \pm 6.4 *(35-66)$ \\
Total arousal index & $22.0 \pm 13.1(6-52)$ & $10.5 \pm 4.2 *(5-21)$ \\
$\quad(/ \mathrm{h} \mathrm{TST})$ & & \\
\hline
\end{tabular}

$* p<0.005$. Comparisons of variables according to group assignment were made with independent $t$ tests or $\chi^{2}$ analyses with Fisher's exact test (dichotomous outcomes). Ranges are provided in parentheses. BMI, body mass index; OAHI, obstructive apnea-hypopnea index; $\mathrm{PEtCO}_{2}$, end-tidal carbon dioxide. 


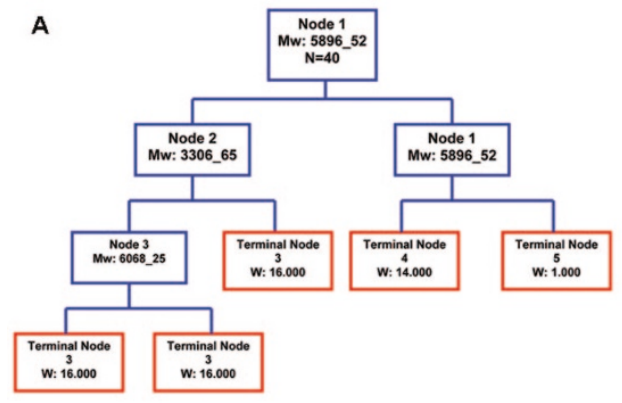

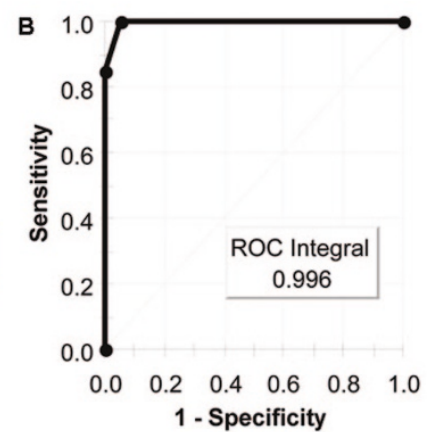

Figure 1. (A) Diagram of a single best decision tree showing the molecular mass of the biomarker proteins and total number of samples. (B) Graph showing the sensitivity and specificity of the discovered biomarker protein with 0.996 receiver operating characteristic (ROC) integral level.
Table 2. Decision tree analysis of proteomic spectral patterns during the learning and test phases

\begin{tabular}{ccrrr}
\hline & & & & \\
& $\mathrm{n}$ & \% correct & OSA & HS \\
\hline Learn & & & & \\
OSA & 18 & 94.444 & 17 & 1 \\
HS & 20 & 100.000 & 0 & 20 \\
Test & & & & \\
OSA & 18 & 93.333 & 15 & 3 \\
HS & 20 & 90.000 & 2 & 18 \\
\hline
\end{tabular}

Swiss-prot database search with the ranges: molecular mass $=$ 5896, $\mathrm{pI}=4$, delta $\mathrm{pI}=1.00$, delta molecular mass $=2 \%$, organism species (OS) or organism classification (OC) human. We found eight proteins in the database in the specified $\mathrm{pI} /$ molecular mass ranges. Of the eight candidate proteins, seven were from the family of Gag polyproteins belonging to the human immunodeficiency virus (HIV). The chance of the identity of this protein being a HIV protein present in serum samples from almost all OSA children and being absent in all non-OSA subjects is highly unlikely. The most likely candidate is osteocalcin precursor (gamma-carboxyglutamic acid containing protein) with a molecular mass of 5799.49 and a pI 4.40. This protein constitutes $1 \%$ to $2 \%$ of the total bone protein. It binds strongly to apatite and calcium. The calcium binding requires gamma-carboxyglutamate residues that are formed by vitamin $\mathrm{K}$-dependent carboxylation (22).

\section{DISCUSSION}

The major findings of the present study indicate that among snoring children, those with clearly defined sleep-disordered breathing and who require referral for treatment can be accurately differentiated using the differential expression of a selected number of proteins in their morning serum samples from snoring children who are not currently considered as candidates for therapy (23).

Before we discuss the potential significance of our findings, some technical issues deserve comment. First, serum samples have been previously used in the discovery process of novel biomarkers using protein mass spectrometry techniques (2427). Indeed, SELDI approaches offer a sensitive alternative to two-dimensional polyacrylamide gel electrophoresis for discovering of disease-associated proteins. The process of SELDI involves purified or partially purified proteins mixed with
EAMs, a crystal-forming matrix, placed on an inert metal target, and subjected to a pulsed laser beam to produce gas phase ions that traverse a field-free flight tube and then are separated according to their mass-dependent velocities (28). Particular precautions were taken to standardize the timing of serum collection and the demographic characteristics of the study groups. Indeed, the two groups did not differ in age, gender, or body mass index. Furthermore, we specifically selected snoring children who fulfilled the currently accepted criteria for surgical removal of tonsils and adenoids and compared them with children who, despite having habitual snoring and being referred for evaluation by their primary care physicians, would not be viewed as requiring any therapeutic intervention based on their polysomnographic findings. We believe that this approach is more realistic and appropriate in the usual clinical contextual setting than using asymptomatic, nonsnoring controls. Therefore, the current findings should be highly relevant to the identification of snoring children who have sleep-disordered breathing and who require more extensive evaluation and treatment. Finally, the software-driven approach taken for tentative identification of the proteins corresponding to the peaks associated with predictive value in OSA is clearly only an initial step in such efforts and will require more extensive affinity-based column purification of large sample volumes of pooled serum samples followed by tandem mass spectrometry (29).

The analytical algorithms used to detect selective patterns of serum protein expression were capable of reliably differentiating between OSA and HS while using a restricted number of spectral peaks. Obviously, verification of the validity of such approach will require prospective and unbiased testing of a large cohort of snoring children with and without the diagnosis of sleep-disordered breathing in several pediatric sleep centers. Such study is clearly beyond the scope of the current work. However, our current findings confirm the initial hypothesis that OSA, possibly via altered gas exchange and sleep fragmentation, leads to alteration of a selective group of proteins in the morning serum and that such proteins can then be potentially used as a diagnostic biomarker for the disease. We have previously shown that serum levels of vascular endothelial growth factor are increased in pediatric patients with sleep apnea and that the magnitude of such increase correlates with disease severity (30). However, use of this single protein as a biomarker was ineffective, with no improvement in the likelihood ratios of diagnosing OSA compared with history and physical examination alone (30). 

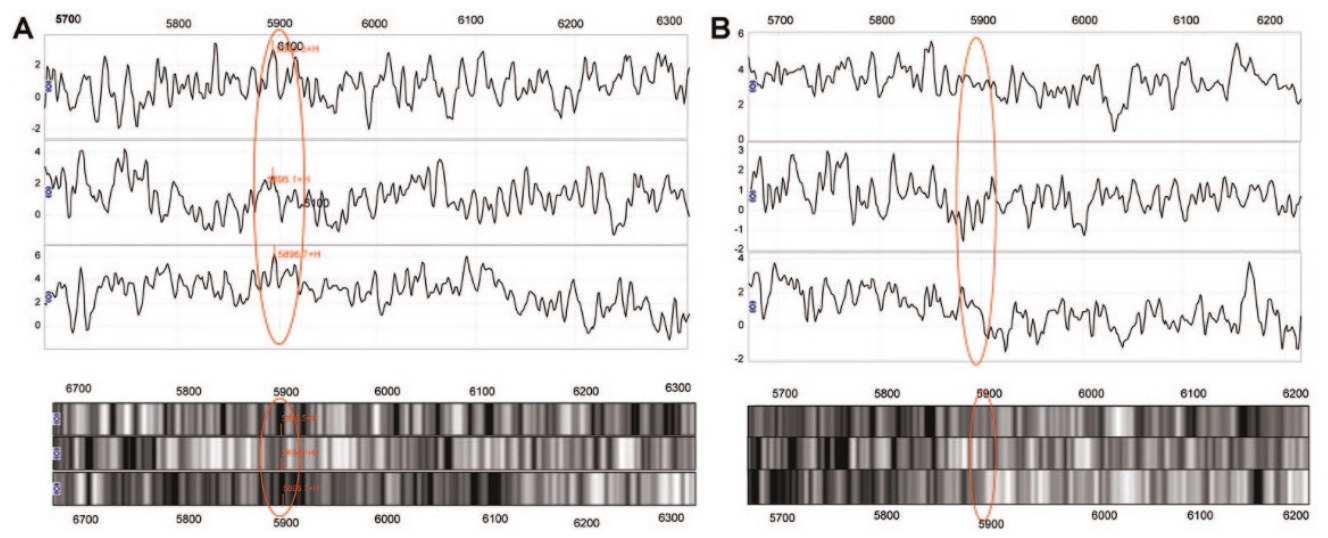

Figure 2. Representative spectra from the serum of three children with OSA $(A)$ and from three children with habitual snoring $(B)$. A peak is clearly recognizable at a molecular mass $5900 \mathrm{Da}$ in all OSA subjects and is absent in all HS children.

Of the three proteins identified in our analysis, we further analyzed the putative and clearly visible biomarker discovered at $\mathrm{m} / \mathrm{z} 5896$ using the Swiss-Prot database. This approach, which constitutes a preliminary and tentative biodiscovery exercise, suggested that a likely identity of this OSA-regulated spectrum may correspond to osteocalcin. Osteocalcin is a precursor of gamma-carboxyglutamic acid-containing protein and is also known as bone Gla protein. This protein is an important marker of bone turnover in physiologic and pathologic conditions (31) and has been widely studied as a biomarker for bone loss, growth retardation (32), osteosarcoma (33), and renal osteodystrophy (34). Previous studies have shown increased serum osteocalcin in diseases with increased bone turnover, e.g. renal osteodystrophy, hyperparathyroidism, hyperthyroidism, and Paget's disease $(35,36)$ and decreased concentrations with low bone turnover, e.g. hypoparathyroidism, hypercalcemia resulting from bone metastases, and longterm corticosteroid therapy, the latter reflecting a decrease in the osteoblastic activity (37). While the role of osteocalcin changes in children with sleep-disordered breathing remains to be established, Colle and colleagues (38) identified increased nocturnal osteocalcin levels among children with growth retardation, possibly as a compensatory response. There is now a substantial body of evidence supporting a causal relationship between stunted somatic growth and OSA in children. Indeed, growth retardation is a frequent observation in children with OSA, even when they are obese (39) and has been attributed to increased energy expenditure during sleep $(40,41)$ as well as to alterations in the regulation of insulin growth factor and its binding proteins (42). In the present study, we also observed that osteocalcin was consistently increased in serum of OSA patients, and this increase could reflect a compensatory phenomenon to the growth deceleration imposed by the episodic asphyxic events associated with recurrent upper airway obstruction. Of note, use of alternative search strategies for the three putative candidate biomarker peaks yielded several potential proteins whose function or expression appears to be regulated by stress. Examples of such candidates include corticotropin, lipotropin, and melanotropin. Obviously, extensive alternative techniques such as LC-MS-MS approaches and greatly expanded number of subjects will be needed to definitively identify these proteins as disease-related biomarkers and their roles in OSA.

In conclusion, proteomic profiling of serum samples in children with OSA revealed differential expression of circulating proteins that may provide useful future diagnostic approaches. Indeed, while prospective, large-scale studies will be required to confirm whether the currently discovered proteomic pattern can dependably differentiate between HS and OSA, such an approach coupled with definitive identification of the differentially expressed proteins will provide the opportunity for developing clinically applicable antibody-based diagnostic assays in pediatric OSA.

\section{REFERENCES}

1. Ali NJ, Pitson DJ, Stradling JR 1993 Snoring, sleep disturbance, and behaviour in 4-5 year olds. Arch Dis Child 68:360-366

2. Gislason T, Benediktsdottir B 1995 Snoring, apneic episodes, and nocturnal hypoxemia among children 6 months to 6 years old. An epidemiologic study of lower limit of prevalence. Chest 107:963-966

3. Nieto FJ, Young TB, Lind BK, Shahar E, Samet JM, Redline S, D'Agostino RB, Newman AB, Lebowitz MD, Pickering TG 2000 Association of sleep-disordered breathing, sleep apnea, and hypertension in a large community-based study. Sleep Heart Health Study. JAMA 283:1829-1836

4. Teculescu DB, Caillier I, Perrin P, Rebstock E, Rauch A 1992 Snoring in French preschool children. Pediatr Pulmonol 13:239-244

5. Hultcrantz E, Lofstrand-Tidestrom B, Ahlquist-Rastad J 1995 The epidemiology of sleep related breathing disorder in children. Int J Pediatr Otorhinolaryngol 32 Suppl:S63-S66.

6. Owen GO, Canter RJ, Robinson A 1996 Snoring, apnoea and ENT symptoms in the paediatric community. Clin Otolaryngology 21:130-134

7. Ferreira AM, Clemente V, Gozal D, Gomes A, Pissarra C, Cesar H, Coelho I, Silva $\mathrm{CF}$, Azevedo MH 2000 Snoring in Portuguese primary school children. Pediatrics 106:E64

8. O'Brien LM, Holbrook CR, Mervis CB, Klaus CJ, Bruner JL, Raffield TJ, Rutherford J, Mehl RC, Wang M, Tuell A, Hume BC, Gozal D 2003 Sleep and neurobehavioral characteristics of 5- to 7-year-old children with parentally reported symptoms of attention-deficit/hyperactivity disorder. Pediatrics 111:554-563

9. Carroll JL, McColley SA, Marcus CL, Curtis S, Loughlin GM 1995 Inability of clinical history to distinguish primary snoring from obstructive sleep apnea syndrome in children. Chest 108:610-618

10. Kang X, Xu Y, Wu X, Liang Y, Wang C, Guo J, Wang Y, Chen M, Wu D, Wang Y, Bi S, Qiu Y, Lu P, Cheng J, Xiao B, Hu L, Gao X, Liu J, Wang Y, Song Y, Zhang L, Suo F, Chen T, Huang Z, Zhao Y, Lu H, Pan C, Tang H 2005 Proteomic fingerprints for potential application to early diagnosis of severe acute respiratory syndrome. Clin Chem 51:56-64

11. Li J, Zhang Z, Rosenzweig J, Wang YY, Chan DW 2002 Proteomics and bioinformatics approaches for identification of serum biomarkers to detect breast cancer. Clin Chem 48:1296-1304

12. Merchant M, Weinberger SR 2000 Recent advancements in surface-enhanced laser desorption/ionization-time of flight-mass spectrometry. Electrophoresis 21:1164 1177

13. Kuwata H, Yip TT, Yip CL, Tomita M, Hutchens TW 1998 Bactericidal domain of lactoferrin: detection, quantitation, and characterization of lactoferricin in serum by SELDI affinity mass spectrometry. Biochem Biophys Res Commun 245:764-773 
14. Qu Y, Adam BL, Yasui Y, Ward MD, Cazares LH, Schellhammer PF, Feng Z, Semmes OJ, Wright GL Jr 2002 Boosted decision tree analysis of surface-enhanced laser desorption/ionization mass spectral serum profiles discriminates prostate cancer from noncancer patients. Clin Chem 48:1835-1843

15. Adam BL, Qu Y, Davis JW, Ward MD, Clements MA, Cazares LH, Semmes OJ, Schellhammer PF, Yasui Y, Feng Z, Wright GL Jr 2002 Serum protein fingerprinting coupled with a pattern-matching algorithm distinguishes prostate cancer from benign prostate hyperplasia and healthy men. Cancer Res 62:3609-3614

16. Petricoin EF, Ardekani AM, Hitt BA, Levine PJ, Fusaro VA, Steinberg SM, Mills GB, Simone C, Fishman DA, Kohn EC, Liotta LA 2002 Use of proteomic patterns in serum to identify ovarian cancer. Lancet 359:572-577

17. National Institutes of Health Rechtschaffen A, Kales AA (eds) 1968 A manual of standardized terminology, techniques and scoring systems for sleep stages of human subject. Government Printing Office, Washington DC, No. 204.

18. Marcus CL, Omlin KJ, Basinki DJ, Bailey SL, Rachal AB, Von Pechmann WS, Keens TG, Ward SL 1992 Normal polysomnographic values for children and adolescents. Am Rev Respir Dis 146:1235-1239

19. 1996 Standards and indications for cardiopulmonary sleep studies in children. American Thoracic Society. Am J Respir Crit Care Med 153:866-878

20. 1992 EEG arousals: scoring rules and examples: a preliminary report from the Sleep Disorders Atlas Task Force of the American Sleep Disorders Association. Sleep 15:173-184

21. Mograss MA, Ducharme FM, Brouillette RT 1994 Movement/arousals. Description, classification, and relationship to sleep apnea in children. Am J Respir Crit Care Med 150:1690-1696

22. Poser JW, Esch FS, Ling NC, Price PA 1980 Isolation and sequence of the vitamin $\mathrm{K}$-dependent protein from human bone. Undercarboxylation of the first glutamic acid residue. J Biol Chem 255:8685-8691

23. Section on Pediatric Pulmonology. Subcommittee on Obstructive Sleep Apnea Syndrome. American Academy of Pediatrics 2002 Clinical practice guideline: diagnosis and management of childhood obstructive sleep apnea syndrome. Pediatrics 109:704-712

24. Paradis V, Degos F, Dargere D, Pham N, Belghiti J, Degott C, Janeau JL, Bezeaud A, Delforge D, Cubizolles M, Laurendeau I, Bedossa P 2005 Identification of a new marker of hepatocellular carcinoma by serum protein profiling of patients with chronic liver diseases. Hepatology 41:40-47

25. Chen YD, Zheng S, Yu JK, Hu X 2004 Artificial neural networks analysis of surface-enhanced laser desorption/ionization mass spectra of serum protein pattern distinguishes colorectal cancer from healthy population. Clin Cancer Res 10:83808385

26. Ebert MP, Meuer J, Wiemer JC, Schulz HU, Reymond MA, Traugott U, Malfertheiner P, Rocken C 2004 Identification of gastric cancer patients by serum protein profiling. J Proteome Res 3:1261-1266

27. Becker S, Cazares LH, Watson P, Lynch H, Semmes OJ, Drake RR, Laronga C 2004 Surfaced-enhanced laser desorption/ionization time-of-flight (SELDI-TOF) differ- entiation of serum protein profiles of BRCA-1 and sporadic breast cancer. Ann Surg Oncol 11:907-914

28. Li J, Zhang Z, Rosenzweig J, Wang YY, Chan DW 2002 Proteomics and bioinformatics approaches for identification of serum biomarkers to detect breast cancer. Clin Chem 48:1296-1304

29. Bischoff R, Luider TM 2004 Methodological advances in the discovery of protein and peptide disease markers. J Chromatogr B Analyt Technol Biomed Life Sci 803:27-40

30. Gozal D, Lipton AJ, Jones KL 2002 Circulating vascular endothelial growth factor levels in patients with obstructive sleep apnea. Sleep 25:59-65

31. Cioffi M, Molinari AM, Gazzerro P, Di Finizio B, Fratta M, Deufemia A, Puca GA 1997 Serum osteocalcin in 1634 healthy children. Clin Chem 43:543-545

32. Ambroszkiewicz J, Gajewska J, Laskowska-Klita T 2002 [Serum osteocalcin and bone alkaline phosphatase in healthy children in relation to age and gender]. Med Wieku Rozwoj 6:257-265

33. Ferrari S, Zolezzi C, Pratelli L, Fasano MC, Bacci G 2003 Urinary excretion of pyridinium cross-links and serum osteocalcin levels in patients with primary highgrade osteosarcoma. Calcif Tissue Int 73:1-4

34. Baskin E, Besbas N, Saatci U, Hascelik G, Topaloglu R, Ozen S, Bakkaloglu A 2004 Biochemical markers of bone turnover in the diagnosis of renal osteodystrophy in dialyzed children. Turk J Pediatr 46:28-31

35. Leger J, Thizon de Gaulle I, Czernichow P 1993 Bone demineralization and elevation of serum osteocalcin concentrations in young children with hyperthyroidism. [Article in French] Ann Pediatr (Paris) 40:404-409

36. Takami H, Shikata J 1990 Serum bone Gla protein as an indicator of parathyroidectomy in patients with secondary hyperparathyroidism. World J Surg $14: 431-435$

37. Meeran K, Hattersley A, Burrin J, Shiner R, Ibbertson K 1995 Oral and inhaled corticosteroids reduce bone formation as shown by plasma osteocalcin levels. Am J Respir Crit Care Med 151:333-336

38. Colle M, Ruffie A, Ruedas E, Chebbo M 1987 Osteocalcin in children of short stature and its nocturnal variations. Arch Fr Pediatr 44:839-841

39. Soultan Z, Wadowski S, Rao M, Kravath RE 1999 Effect of treating obstructive sleep apnea by tonsillectomy and/or adenoidectomy on obesity in children. Arch Pediatr Adolesc Med 153:33-37

40. Marcus CL, Carroll JL, Koerner CB, Hamer A, Lutz J, Loughlin GM 1994 Determinants of growth in children with the obstructive sleep apnea syndrome. J Pediatr 125:556-562

41. Bland RM, Bulgarelli S, Ventham JC, Jackson D, Reilly JJ, Paton JY 2001 Total energy expenditure in children with obstructive sleep apnoea syndrome. Eur Respir J 18:164-169

42. Bar A, Tarasiuk A, Segev Y, Phillip M, Tal A 1999 The effect of adenotonsillectomy on serum insulin-like growth factor-I and growth in children with obstructive sleep apnea syndrome. J Pediatr 135:76-80 